\title{
Inhalt
}

Henning Lobin/Andreas Witt/Angelika Wöllstein (unter Mitarbeit von Harald Lüngen)

Deutsch in Europa - sprachpolitisch, grammatisch, methodisch - XI

\section{Sprach(en)politik in Europa}

Johannes Ebert

Per Anhalter durch die Deutsch-Galaxis: Zur Situation der deutschen Sprache in Europa - 3

Vít Dovalil

Deutsch als Sprachmanagement-Objekt in Europa: Akteure, Interessen und Hindernisse -17

Rahel Beyer/Albrecht Plewnia

Über Grenzen. Deutschsprachige Minderheiten in Europa — 35

Marina Foschi Albert

Deutsch als Fremdsprache in Europa: das „Ökosystem“ Germanistik und seine Nachhaltigkeit -55

\section{Erwerb, Konvergenzen, Divergenzen und Wandel des} Deutschen im Europäischen Kontext

Natalia Gagarina/Sophia Czapka/Nathalie Topaj/Manfred Krifka

Erwerbsprofile des Deutschen im mehrsprachigen Kontext - 75

Nanna Fuhrhop

Vergleichende Graphematik — 93

Lutz Gunkel/Jutta M. Hartmann

Präpositionalobjektsätze im europäischen Vergleich — 111 
Thomas Stolz/Nataliya Levkovych

Über die Diffusion von / $y$ / in Europa und wie viel Verantwortung das Deutsche dafür trägt 135

Peter Auer

Gibt es einen deutschen Neo-Standard und - wenn ja - wie verhält er sich zu den Entwicklungen der Standards anderer europäischer Sprachen?

\section{Methoden - Sprachressourcen und Infrastrukturen}

Erhard Hinrichs

Multilinguale Sprachressourcen für die linguistische Forschung 189

Beata Trawiński/Marc Kupietz

Von monolingualen Korpora über Parallel- und Vergleichskorpora zum

Europäischen Referenzkorpus EuReCo 209

Anke Lüdeling/Hagen Hirschmann/Anna Shadrova/Shujun Wan

Tiefe Analyse von Lernerkorpora — 235

\section{Methodenmesse}

Nils Diewald/Franck Bodmer/Peter Harders/Elena Irimia/Marc Kupietz/Eliza Margaretha/Helge Stallkamp

KORAP und EURECo - Recherchieren in mehrsprachigen vergleichbaren Korpora -287

Stefan Falke/Saskia Ripp/Roman Schneider/Ulrich Hermann Waßner Das Informationssystem grammis als Ressource für die internationale Germanistik 295

Beata Trawiński/Susan Schlotthauer/Piotr Bański

CoMParS: Eine Sammlung von multilingualen Parallelsequenzen des Deutschen und anderer europäischer Sprachen — 301

Tomáš Káňa

InterCorp: viele Sprachen - ein Korpus

Ein multilinguales Parallelkorpus (nicht nur) europäischer Sprachen — 311 
Andreas Nolda/Adrien Barbaresi/Alexander Geyken

Das ZDL-Regionalkorpus: Ein Korpus für die lexikografische Beschreibung der diatopischen Variation im Standarddeutschen - 317

Erhard Hinrichs/Patricia Fischer/Yana Strakatova

Rover und TüNDRA: Such- und Visualisierungsplattformen für Wortnetze und Baumbanken - 323

Antonina Werthmann/Andreas Witt/Jutta Bopp

Verbundprojekt CLARIAH-DE - Eine nachhaltige Forschungsinfrastruktur für die Geistes-, Kultur- und Sozialwissenschaften 329 
